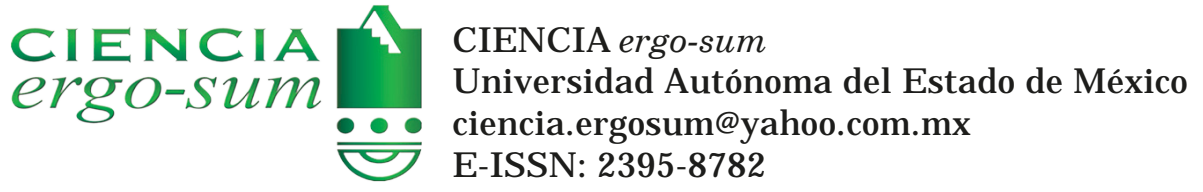

\title{
Microcrédito y desarrollo de las microempresas en las zonas rurales de Ancash, Perú
}

Maguiña Palma, Misael Erikson; Ramírez Asís, E dwin Hernán; Huerta Soto, Rosario Mercedes; Concepción Lázaro, RobertoJ esús

Microcrédito y desarrollo de las microempresas en las zonas rurales de Ancash, Perú

CIENCIA ergo-sum, vol. 28, núm. 1, marzo-junio 2021|e109

Universidad Autónoma del Estado de México, México

Esta obra está bajo una Licencia Creative Commons Atribución-NoComercial-SinDerivar 4.0 Internacional .

Maguiña Palma, M. E., Ramírez Asís, E. H., Huerta Soto, R. M. y Concepción Lázaro, R. J . (2020). Microcrédito y desarrollo de las microempresas en las zonas rurales de Ancash, Perú. CIE NCIA ergo-sum, 28(1). https://doi. org/10.30878/ces.v28n1a3 


\title{
Microcrédito y desarrollo de las microempresas en las zonas rurales de Ancash, Perú
}

\section{Micro-credit and micro-enterprise development in Ancash rural areas, Peru}

\author{
Misael Erikson Maguiña Palma \\ Universidad Norbert Wiener, Perú \\ mmaguia@hotmail.com \\ (1) https://orcid.org/0000-0002-4545-7413 \\ Edwin Hernán Ramírez Asis \\ Universidad Nacional Santiago Antúnez de Mayolo, Perú \\ ehramireza@unasam.edu.pe \\ (D) https://orcid.org/0000-0002-9918-7607 \\ Rosario Mercedes Huerta Soto \\ Universidad Nacional Santiago Antúnez de Mayolo, Perú \\ mhuertas@unasam.edu.pe \\ (D) https://orcid.org/0000-0002-1738-3437 \\ Roberto Jesús Concepción Lázaro \\ Universidad San Pedro, Perú \\ conc7000@gmail.com \\ (1) https://orcid.org/0000-0003-1996-7767
}

\author{
Recepción: 13 de junio de 2019 \\ Aprobación: 01 de octubre de 2019
}

\section{Resumen}

El microcrédito implica otorgar pequeños préstamos con la esperanza de lograr el desarrollo de unidades de negocio mediante instituciones financieras en la población periférica peruana. Dentro de este contexto, se busca determinar la relación del microcrédito y el desarrollo de las microempresas en las zonas rurales. En este sentido, se entrevistaron a 278 microempresarias y el instrumento de recolección de datos fue sometido a una validez por juicios de expertos con un coeficiente de acuerdos de $97 \%$ y consistencia interna alfa de Cronbach de 0.90. Los resultados obtenidos muestran que el microcrédito Súper Mujer tiene una relación directa y significativa con el desarrollo de las microempresas en las zonas rurales de acuerdo con el coeficiente de correlación de Spearman (rho 0.84, Sig. 0.000*). Palabras clave: crédito, estrategia de desarrollo, microfinanzas, microempresarias, rural.

\begin{abstract}
Abstrac
The micro-credit implies the granting of small loans in the hope of achieving the development of business units through financial institutions in the peripheral Peruvian population. In this context, the objective of the article is to determine the relationship between micro-credit and the development of micro-enterprises in rural areas. 278 women micro-entrepreneurs were interviewed, the data collection instrument was subjected to a validity by expert judgments with a coefficient of agreement of $97 \%$ and Cronbach's internal alpha consistency of 0.90 . The results obtained show that the super woman's micro-credit has a direct and significant relationship with the development of micro-enterprises in rural areas according to the Spearman's Correlation coefficient (rho 0.84, Sig. 0.000*).
\end{abstract}

KEYWORDS: Credit, development strategy, microfinance, micro-entrepreneurs, rural.

\section{INTRODUCCIÓN}

La región Ancash se localiza al oeste del Perú: limita por el norte con La Libertad, al sur con la región Lima, al este con la región Huánuco y por el oeste con el Océano Pacífico. Está ubicada a una altitud media de 2688 msnm; 
incluye las provincias de Caraz, Yungay, Carhuaz y Huaraz. Con una población de 10900 habitantes, es la octava región más poblada y la sexta economía del Perú por contribuir al Producto Bruto Interno en $3.5 \%$.

Nurske (1953) instauró un paradigma donde manifiesta que los pobres no pueden salir de su situación porque el total de sus ingresos lo destinan a cubrir sus necesidades primarias, lo que les impide ahorrar e invertir; sin embargo, según Hamad y Fernald (2015) han reportado exitosos programas de microcréditos en mujeres, cuyos resultados contribuyen a que se puedan tomar mejores decisiones financieras si la administración del dinero es por parte de ellas, y mejora a su vez su posición en la comunidad.

Los primeros bancos comunales fueron establecidos en la India y fueron dirigidos por Muhammad Yunnus, quien tuvo la gran idea de fundar el Grameen Bank (Rural Bank), el cual estaba dirigido a aquellas mujeres humildes que se organizaban entre cinco para poder acceder a un pequeño crédito (FINCA, 2019); el modelo original de banca comunal se ha adaptado de acuerdo con las necesidades particulares y el entorno del territorio que se va a servir. La naturaleza de la banca comunal permanece en su base central, que consiste en brindar servicios financieros a los más pobres a través del establecimiento de asociaciones de personas formadas para proporcionar créditos a todos de sus miembros y alentarlos en la cultura del ahorro (Siccha, 2017). En efecto, más allá de la inclusión financiera de las personas, los bancos comunales lidian con las necesidades de la sociedad, en especial para los sectores más vulnerables al autogestionar, apoyar y empoderar a sus clientes en el ámbito económico, político y social (Peñafiel Valarezo y Valle Vera, 2018).

El microcrédito es un medio para incrementar el acceso al financiamiento creado como propuesta para superar las restricciones del sistema financiero formal para miles de familias que tienen un negocio. Su esencia está en que todos puedan tener acceso al recurso económico en mejores condiciones (Lacalle, 2008). Según Duran (2010), en África, mediante los programas de microcréditos se ha promovido el acceso al financiamiento a mujeres en la región gracias a los bancos comunales, y se han obteniendo resultados favorables para las beneficiarias. En el Perú, entre los años 1990 y 2005, la ONG Prisma tuvo su participación con el modelo de bancos comunales Asociación Comunal para el Desarrollo (ACPD); sus principales intervenciones se dieron en Lima y algunas provincias del Perú. Entre sus principales logros se encuentra el de insertar a $70 \%$ de sus clientes al sistema financiero por la experiencia obtenida con este tipo de microcrédito, pero no pudo continuar debido a la falta de financiamiento (Pilares, 2016).

Las microempresarias, en su mayoría, en las zonas rurales de Ancash poseen un negocio ambulatorio y otras concentran sus actividades en restaurantes, peluquerías, servicios de internet, pequeñas tiendas y dulcerías, entre otros (Ramírez, Maguiña y Huerta, 2020). El impacto económico en las microempresas es poco visible, ya que sus ingresos son destinados para cubrir deudas y gastos familiares.

Mibanco y la Caja Municipal del Santa tienen oficinas en Ancash, donde ofrecen créditos individuales para microempresarios que tengan negocios formales e informales con una experiencia de seis meses. Además, Compartamos Financiera es la única que ofrece el producto de banca comunal denominado microcrédito Súper Mujer y dirigida exclusivamente a mujeres microempresarias cuyas actividades económicas son el comercio y el servicio (SBS, 2019). Compartamos Financiera busca crear valor económico, social y humano mediante su producto de banca comunal, siendo la intermediaria de microfinanzas más representativa en el Perú con más de 20 años de permanencia en el mercado. La misión de la empresa es generar mejores oportunidades para las mujeres el desarrollo de sus microempresas brindando acceso a servicios financieros en condiciones de accesibilidad (Torre et al., 2012).

Los montos de los microcréditos se otorgan inicialmente hasta 285 USD a ser pagados en 4 meses, con una frecuencia de pago catorcenal y con una tasa de interés efectiva anual (TEA) dividida en tres modelos: bronce (136.85\%), plata (125.22\%) y oro (101.22\%). Los pagos catorcenales no son bien vistos por las clientas porque se les dificulta conseguir las cuotas en tan poco tiempo, ya que las actividades económicas de algunas de ellas no brindan ingresos tan frecuentemente como las clientas que se dedican a la agricultura y a la ganadería. Asimismo, el microcrédito Súper Mujer brinda beneficios como a) la educación financiera, que consiste en brindar charlas informativas sobre cómo mantener el crecimiento de su negocio, $b$ ) el seguro de desgravamen, que condona la 
deuda del crédito en caso de fallecimiento de la clienta previa evolución del comportamiento del grupo, $c$ ) atención personalizada, la cual brinda la información oportuna requerida por las clientas, y d) crédito de fácil acceso, porque es un crédito de confianza y de requisitos mínimos como recibo de servicios (agua o luz) y copia del Documento Nacional de Identidad (Siccha, 2017). En consecuencia, existe la necesidad de realizar un análisis y explicar la relación entre el microcrédito Súper Mujer y el desarrollo de las microempresas en las zonas rurales de Ancash del Perú.

\section{Métodos y materiales}

El estudio fue descriptivo-correlacional, con un diseño no experimental transversal. La técnica empleada en este estudio fue la encuesta. El cuestionario fue validado mediante juicio de cinco expertos ( $p$-valué $0.01^{*}$, el coeficiente de acuerdos de 97\%) y consistencia interna (alfa de Cronbach $\geq 85 \%$ ).

La muestra estuvo constituida por 278 clientas de las provincias Huaraz, Carhuaz y Yungay, que accedieron al microcrédito Súper Mujer de Compartamos Financiera.

La prueba de normalidad (Kolmogorov-Smirnov) identificó que las variables del estudio se ajustaron a una distribución normal. Por ello, se utilizó el estadístico de correlación de Spearman (rho) para contrastar la hipótesis de investigación.

\section{Resultados}

Se puede observar que la mayoría de las clientas califican al microcrédito como regular y al desarrollo de sus microempresas como rentable; esto quiere decir que el efecto que genera el uso del microcrédito todavía es moderado. Debemos resaltar que existe un $18 \%$ que tiene una percepción positiva del desarrollo de su microempresa (cuadro 1).

Las clientas que tienen un crédito de monto bajo son las que califican el desarrollo de las microempresas de rentable; por otro lado, sólo $11 \%$ de las clientas tiene créditos mayores a 631 USD (cuadro 2).

\section{CUADRO 1}

Microcrédito y desarrollo de las microempresas

\begin{tabular}{lrrrrrrrr}
\hline \multirow{2}{*}{$\begin{array}{l}\text { Microcrédito } \\
\text { Súper Mujer }\end{array}$} & \multicolumn{9}{c}{ Poco rentable } & \multicolumn{2}{c}{ Rentable } & \multicolumn{2}{c}{ Muy rentable } & \multicolumn{2}{c}{ Total } \\
& $\mathrm{N}$ & $\%$ & $\mathrm{~N}$ & \multicolumn{1}{c}{$\%$} & $\mathrm{~N}$ & $\%$ & $\mathrm{~N}$ & $\%$ \\
\hline Malo & 42 & $15 \%$ & 21 & $8 \%$ & 0 & $0 \%$ & 63 & $23 \%$ \\
Regular & 0 & $0 \%$ & 131 & $47 \%$ & 34 & $12 \%$ & 165 & $59 \%$ \\
Bueno & 0 & $0 \%$ & 0 & $0 \%$ & 50 & $18 \%$ & 50 & $18 \%$ \\
Total & 42 & $15 \%$ & 152 & $55 \%$ & 84 & $30 \%$ & 278 & $100 \%$ \\
\hline
\end{tabular}

En su mayoría las microempresarias perciben el ahorro generado por el microcrédito en un nivel regular y opinan que el desarrollo de las microempresas es rentable. Además, 30\% de las clientas considera el ahorro generado como alto y un desarrollo de las microempresas de muy rentable. De acuerdo con lo anterior, quiere decir que a mayor ahorro mejor es la percepción de los resultados generados por en su microempresa (cuadro 3).

La relación de la variable Desarrollo de la Microempresa y las dimensiones Monto del Crédito (rho 0.82 y el $p$-valor $0.001^{*}$ ), Frecuencia de pago (rho 0.85 y el $p$-valor $0.002^{*}$ ) y Ahorro generado (rho 0.73 y el $p$-valor $\left.0.006^{*}\right)$ son directas. En cuanto a las variables Microcrédito y Desarrollo de la microempresa la relación es positiva y significativa (rho 0.84 y el $p$-valor $0.00^{*}$ ); es decir, la mejora de la percepción del microcrédito tendrá una mejora en la percepción sobre el desarrollo de las microempresas en las zonas rurales de Ancash, Perú. 


\section{CUADRO 2}

Monto del microcrédito y el desarrollo de las microempresas

\begin{tabular}{|c|c|c|c|c|c|c|c|c|}
\hline \multirow{3}{*}{$\begin{array}{l}\text { Monto del microcrédito } \\
\text { USD }\end{array}$} & \multicolumn{6}{|c|}{ Desarrollo de las microempresas } & \multirow{2}{*}{\multicolumn{2}{|c|}{ Total }} \\
\hline & \multicolumn{2}{|c|}{ Poco rentable } & \multicolumn{2}{|c|}{ Rentable } & \multicolumn{2}{|c|}{ Muy rentable } & & \\
\hline & $\mathrm{N}$ & $\%$ & $\mathrm{~N}$ & $\%$ & $\mathrm{~N}$ & $\%$ & $\mathrm{~N}$ & $\%$ \\
\hline 230 a 630 & 42 & $15 \%$ & 152 & $55 \%$ & 54 & $19 \%$ & 248 & $89 \%$ \\
\hline 631 a 1020 & 0 & $0 \%$ & 0 & $0 \%$ & 24 & $9 \%$ & 24 & $9 \%$ \\
\hline 1021 a 1430 & 0 & $0 \%$ & 0 & $0 \%$ & 6 & $2 \%$ & 6 & $2 \%$ \\
\hline Total & 42 & $15 \%$ & 152 & $55 \%$ & 84 & $30 \%$ & 278 & $100 \%$ \\
\hline
\end{tabular}

Fuente: elaboración propia.

\section{CUADRO 3}

Ahorro generado y desarrollo de las microempresas

\begin{tabular}{|c|c|c|c|c|c|c|c|c|}
\hline \multirow{3}{*}{ Ahorro generado } & \multicolumn{6}{|c|}{ Desarrollo de las microempresas } & \multirow{2}{*}{\multicolumn{2}{|c|}{ Total }} \\
\hline & \multicolumn{2}{|c|}{ Poco rentable } & \multicolumn{2}{|c|}{ Rentable } & \multicolumn{2}{|c|}{ Muy rentable } & & \\
\hline & $\mathrm{N}$ & $\%$ & $\mathrm{~N}$ & $\%$ & $\mathrm{~N}$ & $\%$ & $\mathrm{~N}$ & $\%$ \\
\hline Bajo & 24 & $9 \%$ & 0 & $0 \%$ & 0 & $0 \%$ & 24 & $9 \%$ \\
\hline Regular & 18 & $6 \%$ & 116 & $42 \%$ & 0 & $0 \%$ & 134 & $48 \%$ \\
\hline Alto & 0 & $0 \%$ & 36 & $13 \%$ & 84 & $30 \%$ & 120 & $43 \%$ \\
\hline Total & 42 & $15 \%$ & 152 & $55 \%$ & 84 & $30 \%$ & 278 & $100 \%$ \\
\hline
\end{tabular}

Fuente: elaboración propia.

\section{Discusión}

De acuerdo con la calificación del desarrollo de las microempresas en las zonas rurales de Ancash, 55\% percibe que es rentable y un 15\% como poco rentable; este resultado está por debajo de lo expuesto por Espín (2011), donde afirma que un $82 \%$ indica que su situación económica mejoró, mientras que un mínimo y no importante $4 \%$ de mujeres califica al microcrédito como negativo en la situación económica. Del mismo modo, 53\% de las clientas percibe el microcrédito en un nivel regular y a su vez $47 \%$ considera el desarrollo de las microempresas como rentables. Estas estadísticas mantienen relación con lo que afirma Siccha (2015), donde expresa que 48\% de las microempresarias menciona que el microcrédito ha sido representativo para el desarrollo de su microempresa y un $43 \%$ ha sido muy representativo para el desarrollo de su negocio familiar.

En cuanto a la consolidación de los grupos, se da a conocer a todas las clientas las características, beneficios y condiciones del microcrédito; para esto, se programan reuniones en la preaprobación del microcrédito. Por lo general, los problemas se presentan cuando las clientas no acuden a las reuniones e impiden el progreso de la formación del grupo y la aprobación del crédito al prolongar el tiempo que causarían una disolución. Cuando el crédito está en curso se presentan inconvenientes en algunos grupos por la falta de conocimiento sobre la garantía solidaria; esta situación hace que el grupo entre en mora y perjudique a las clientas que se encuentran bien calificadas en el sistema financiero por el monto total del crédito. Es así como se demuestra que la frecuencia de la garantía solidaria en los grupos se da muy pocas veces. Por otra parte, todos los integrantes del grupo solidario son responsables de los microcréditos de manera asociada, esto es, los miembros presionan a sus integrantes para pagar lo que corresponde al microcrédito (Inglada-Galiana et al., 2015). Por ello, en ciertas ocasiones las mujeres expresan "que son socias de un banco comunal que les brinda apoyo"; se sienten tranquilas porque pueden cubrir sus necesidades y entre las integrantes se genera un ambiente de solidaridad, de ayuda mutua. En la vida diaria suceden eventos como accidentes de tránsito, de trabajo o algún otro suceso inesperado; para atenderlas se recurre a la solidaridad de las asociadas que buscan apoyar en la solución del problema (Ferrer y Ginés, 2010). 
En correlación con los montos del microcrédito Súper Mujer y el desarrollo de las microempresas en las zonas rurales de Ancash, 89\% de las clientas percibe montos entre 240 USD a 670 USD y 55\% de las clientas califica el desarrollo de las microempresas de rentable; el resultado es similar con lo expuesto por Patzi (2011), quien afirma que el empoderamiento percibido por las clientas se origina gracias al servicio de microcrédito, y por ello la mayoría desea permanecer en el programa. Adicionalmente, para Siccha (2017), el microcrédito juega un papel importante en la mejora económica y el entorno familiar de los clientes.

En cuanto a la frecuencia de pago, $53 \%$ de las clientas califica como bueno al microcrédito Súper Mujer y 55\% al desarrollo de las microempresas como rentable. Al respecto, Pilares (2016) menciona que el tiempo del crédito ha repercutido en la mejora económica; además, se verificó que el lapso para realizar la devolución es cambiante y $71 \%$ presenta una mejora económica alta. Contrariamente, Khan y Sulaiman (2015) concluyen que a pesar de un cambio positivo general en los ingresos estos programas de microcréditos muestran evidencias débiles de beneficiar a los miembros de la comunidad del cuartil inferior durante el periodo comprendido. El estudio sugiere que el microcrédito no es beneficioso para todos los segmentos de los pobres; en las zonas rurales de Ancash las clientas manifiestan que casi siempre están de acuerdo con el plazo, pero proponen que la frecuencia de pago pueda incrementarse a treinta días o a cuotas mensuales.

Por otro parte, en la relación entre la tasa de interés del microcrédito y el desarrollo de las microempresas, 53\% de las clientas percibe una tasa de interés mensual entre 2\% a 4\% del microcrédito Súper Mujer y un 55\% califica el desarrollo de las microempresas como rentable, cuyo resultado coincide con lo que manifiesta Espinoza (2015), quien afirma que el grado de satisfacción es alto respecto a la forma de pago seguido de los plazos, y en un menor grado en las tasas de interés con un $26 \%$ que manifiesta estar satisfecho.

Montalvo Corzo et al. (2018) concluyen que el microcrédito no promueve hábitos saludables como el ahorro o la inversión. Sin embargo, el producto microcrédito Súper Mujer sí promueve el ahorro en las clientas que acceden al crédito; por lo tanto, se considera importante este aspecto en un producto de microcrédito. Además, Báez (2014) menciona que el crecimiento del microcrédito per cápita influye de manera positiva en el ahorro per cápita de la población, es decir, que las personas que son prestatarias de microcrédito se benefician al mejorar su capacidad de ahorro y, por consiguiente, de inversión. Este resultado coincide con lo afirmado por el gerente general Gerson Misael Saavedra More y los dos jefes de crédito, Cinthia Lujan Barreto y Darly Daniel Blas Rafael, quienes coinciden en que los ahorros les favorecen en el momento de renovación del microcrédito para obtener mayores montos. En este mismo sentido, Al-Shami et al. (2016) concluyen que el microcrédito permitió a las mujeres acceder al capital financiero, formar sus micro y pequeñas empresas y obtener un ingreso que les ayude a contribuir al gasto del hogar. Aunque el efecto del microcrédito fue parcial, les proporcionó un mejor control de recursos, además de mejorar el poder de negociación y aumentar su autoestima tanto en sus en sus hogares como en la comunidad.

El microcrédito se relaciona con el desarrollo de las microempresas en las zonas rurales de Ancash debido a que presenta un coeficiente de rho de Spearman $=0.84$, que a su vez es positiva y significativa (sig. $\left.0.00^{*}\right)$, por lo que este hallazgo es similar a lo propuesto por González Rodríguez et al. (2017). Sandoval (2010) concluye que el microcrédito influye positivamente en los clientes porque favorece mejoras en sus condiciones de vida. De igual modo, para De Asís (2003), García (2004) y Foschiatto y Stumpo (2006), el microcrédito tiene un importante efecto en las microempresas y el acceso al microcrédito constituye un elemento importante al momento de lograr el desarrollo de los microempresarios. Además, Gutiérrez-Nieto (2005) y Enimu, Eyo y Ajah (2017) afirman que los diversos microcréditos agrícolas son un mecanismo importante para el desarrollo económico y social, y son los determinantes significativos de la reducción de la exclusión social; por otra parte, favorecen la creación de nuevas unidades de negocios locales. Finalmente, para Ghosh (2013) en la India las instituciones microfinancieras con fines de lucro son problemáticas, las cuales deberían ser reguladas, además de utilizar otras estrategias para la inclusión financiera de los pobres y de los pequeños productores. Por el contrario, en Perú las instituciones microfinancieras son reguladas por la Superintendencia de Banca, Seguros y AFPs y se debería trabajar en el mejoramiento de las condiciones del acceso al microcrédito y por ende propiciar una mejor oferta financiera para las zonas rurales. 


\section{AnÁlisis PRospectivo}

De acuerdo con Soler-Tormo y Melián-Navarro (2012), el triunfo de la banca comunal no es necesariamente por el microcrédito, sino por el ahorro que se promueven entre los miembros del grupo y los beneficios que genera, como el empoderamiento de las clientas. En este escenario, y en el ámbito de estudio, existe una única institución financiera que trabaja con el modelo de bancos comunales; por ello, las clientas son quienes lideran el negocio a pesar de que es una microempresa familiar, en especial en las zonas rurales de Ancash donde existe un dominio de los varones bien marcado que todavía no se puede desterrar. La contribución del microcrédito a las microempresas familiares se observa por el aumento a los ingresos en las unidades de negocios y favorece a que puedan consumir productos, utilizar servicios nuevos, adquirir terreno o comprar nuevas razas de ganado que incremente el capital de la familia. Asimismo, respecto al pueblo o al barrio, que es el entorno, incrementa la probabilidad de mejoras empresariales, lo que favorece el incremento de puestos de trabajo y genera en la economía de dichas zonas rurales un efecto positivo.

Finalmente, se debe avanzar respecto a la inclusión financiera y también en mejorar las condiciones de acceso al microcrédito, lo cual sería un aporte destacable para el fortalecimiento de las zonas agrícolas, dado el fenómeno generalizado del éxodo rural en el Perú.

\section{REFERENCIAS}

Al-Shami, S. S. A., Razali, M. M., Majid, I., Rozelan, A., \& Rashid, N. (2016). The effect of microfinance on women's empowerment: Evidence from Malaysia. Asian Journal of Women's Studies, 22(3), 318-337. https:// doi.org/10.1080/12259276.2016.1205378

Báez, S. (2014). Evaluación del impacto del microcrédito en mujeres emprendedoras en Ecuador entre 2007 y 2012: evaluación del nivel de ingresos mensuales, activos, generación de empleo y ahorro per cápita (tesis de bachiller). Quito: Universidad San Francisco de Quito.

De Asís, A. (coord.) (2003). El microcrédito en el Norte de África y Oriente Medio. Madrid: Fundación Promoción Social de la Cultura.

Duran, J. (2010). African NGO's and womanism: Microcredit and self-help. Journal of African American Studies, 14, 171-180. https://doi.org/10.1007/s12111-009-9109-2

Enimu, S., Eyo, E, \& Ajah, E. (2017). Determinants of loan repayment among agricultural microcredit finance group members in Delta state, Nigeria. Financial Innovation, 3(21). 1-12. https://doi.org/10.1186/ s40854-017-0072-y

Espín, J. (2011). Impacto de las microfinanzas en la economía de la mujer (tesis de maestría). Quito: Universidad Tecnológica Equinoccial.

Espinoza, M. (2015). El microcrédito como motor de desarrollo de la microempresa en Guayaquil. Periodo 20102012 (tesis de maestría). Guayaquil: Universidad de Guayaquil.

Ferrer,J. y Ginés, M. (2010). Experiencias internacionales sobre microfinanzas. Castellón de la Plana: KRKEdiciones. FINCA. (2019). FINCA Ecuador. Disponible en http://www.finca.ec/quienes-somos/modelo-de-negocio/

Foschiatto, P. y Stumpo, G. (2006). Politicas municipales de microcrédito: un instrumento para la dinamización de los sistemas productivos locales. Santiago de Chile: Naciones Unidas.

García, J. (2004). Los micropréstamos como instrumentos de desarrollo en el sur: especial referencia al ámbito latinoamericano. Madrid: CIDEAL.

Ghosh, J. (2013). Microfinance and the challenge of financial inclusion for development. Cambridge Journal of Economics, 37, 1203-1219. https://doi.org/1093/cje/bet042 
González Rodríguez, Z., Leos Rodríguez, J. y Zavala Pineda, M. (2017). Impactos de los créditos agropecuarios de Financiera Nacional de Desarrollo en el bienestar de los hogares beneficiarios del valle de Mexicali. CIENCIA ergo-sum, 24(1), 5-17.

Gutiérrez-Nieto, B. (2005). Antecedentes del microcrédito. Lecciones del pasado para las experiencias actuales. Revista de Economia Pública, Social y Cooperativa, 51, 25-50.

Hamad, R., \& Fernald, L. (2015). Microcredit participation and women's health: Results from a cross-sectional study in Peru. International Journal for Equity in Health, 14(62). 1-10. https://doi.org/10.1186/s12939-015-0194-7

Inglada-Galiana, M., Sastre-Centeno, J., \& De Miguel-Bilbao, M. (2015). Importancia de los microcréditos como instrumento de financiación en el desarrollo social. Revista Cientifica Guillermo de Ockham, 13(2), 89-100. https://doi.org/10.21500/22563202.2264

Khan, Z., \& Sulaiman, J. (2015). Does microcredit help the poor and financially marginalized communities? Experience of Pakistan. In A. Heshmati, E. Maasoumi \& G. Wan (eds.), Poverty reduction policies and practices in developing Asia, Economic studies in inequality, social exclusion, and Well-Being (pp. 31-52). Singapur: Asian Development Bank. https://doi.org/10.1007/978-981-287-420-7_3

Lacalle, M. (2008). Microcréditos y pobreza: de un sueño al Nobel de la Paz. Madrid: Ediciones Turpial S. A.

Montalvo Corzo, R., Vázquez Parra, J. y Amézquita Zamora, J. (2018). Desigualdad, microcréditos y desarrollo sostenible: un estudio en la Zona Metropolitana de Guadalajara. CIENCIA ergo-sum, 25(1), 1-28.

Nurske, R. (1953). Problemas de formación de capital en lospaises insuficientemente desarrollados. México: Fondo de cultura económica.

Patzi, P. (2011). Microcrédito, alternativa de desarrollo para mujer y su familia (tesis de licenciatura). La Paz: Universidad Mayor San Andrés.

Peñafiel Valarezo, G. D. y Valle Vera, J. J. (2018). Los bancos comunales en la Región 5. Ecuador: Universidad Estatal de Milagro.

Pilares, Y. (2016). Los microcréditos y el desarrollo económico de los microempresarios usuarios de Mibanco en la asociación Pro Vivienda los Proceres del distrito de San Sebastian, Cusco, 2015 (tesis de licenciatura). Cusco: Universidad Andina del Cusco.

Ramírez, E., Maguiña, M., \& Huerta, R. (2020). Attitude, satisfaction and loyalty of customers in Municipal Savings Banks of Peru. Retos Revista de Ciencias de la Administración y Economía, 10(20), 313-325. https:// doi.org/10.17163/ret. n20.2020.08

Sandoval, M. (2010). El microcrédito y su incidencia en el desarrollo socioeconómico del sector microempresarial en la ciudad de Ibarra, provincia de Imbabura (tesis de licenciatura). Ecuador: Universidad Técnica del Norte.

SBS (2019). Informe de estabilidad del sistema financiero. Superintendencia de Banca, Seguros y AFPs del Perú.

Siccha, L. (2015). Análisis del impacto de la calidad de vida de las microempresarias que accedieron al microcrédito con la Asociación Mujeres en Acción-AMA, sucursal Trujillo, durante el periodo 2010-2013 (tesis de licenciatura). Perú: Universidad Nacional de Trujillo.

Siccha, L. (2017). Funciones como instructor de crédito grupales en la institución Compartamos Financiera-Agencia Trujillo (trabajo de suficiencia profesional). Perú: Universidad Nacional de Trujillo.

Soler-Tormo, F. y Melián-Navarro, A. (2012). Cooperativas de crédito y banca social: viejas y nuevas respuestas éticas y solidarias a problemas de siempre. Revesco, 109, 45-80.

Torre, B., Sainz, I., Sanfilippo, S. y López, C. (2012). Guía sobre microcréditos. Madrid: Universidad de Cantabria.

CC BY-NC-ND 